\section{Myoclonic jerks secondary to piperacillin and nafcillin}

\author{
Michael A. Meyer \\ Department of Neurology, Sisters \\ Hospital, Buffalo, NY, USA
}

\begin{abstract}
A 66-year-old male receiving intravenous piperacillin and nafcillin for a post-surgical wound infection developed intermittent myoclonic jerks of all four extremities that disappeared after discontinuation of these two medications. In addition there was a mild yet definite intermittent encephalopathic effect; head computed tomography examination as negative and there was no prior history for seizure or myoclonus. These two beta lactam ring antibiotics are structurally similar to penicillin, which is well known to induce not only myoclonus but also seizure activity by reducing the gamma-aminobutyric acid (GABA) induced inhibitory currents by inducing an open chloride channel block of the GABA type A receptors within the brain. Clinicians need to be fully aware of the potential epileptogenic effects of piperacillin, nafcillin, and related antibiotics.
\end{abstract}

\section{Introduction}

Antibiotics are well known to alter neuronal functioning, with penicillin being the best studied on not only a clinical level but on a basic science level as well. After multiple reports emerged on the probable relation between high dose penicillin and seizure, detailed animal studies emerged that showed toxic doses of penicillin given intravenously could induce myoclonic jerks at the spinal level, and could be abolished by penicillinase administration.

Given the wide spread use of structurally related antibiotics that share the beta lactam ring structure of penicillin, clinicians need to be aware of the potential for secondary neurotoxic effects. This report describes reversible frequent myoclonic jerks of the extremities during intravenous administration of nafcillin with piperacillin.

\section{Case Report}

A 66-year-old male who underwent a repeat knee prosthesis surgery developed pain and swelling at the operative site shortly afterwards with fever and elevation of the white blood count to 13,700 . For the treatment of Staphylococcus warneri isolated from the operative site, intravenous antibiotics were given, with vancomycin started initially at a dose of $1.25 \mathrm{gm}$ q8 hours, but then switched to a combination of Nafcillin 2 grams 18 hours IV, plus piperacillin/tazobactam 4.5 grams q6h IV. Shortly after starting this regimen, the patient noted the onset of random myoclonic jerks of the extremities, which would be an isolated limb but at other times be all four limbs at once as a single isolated myoclonic jerk. In addition, the patient reported something is wrong with my time clock and felt surprised that long periods of time had passed, and also had a spell of getting up to go to the bathroom, and feeling there was a gap in time. Approximately 40 random myoclonic jerks were estimated to occur over the course of each day of the six week course of the nafcillin/piperacillin combination by the patient and his wife, who stayed in the room, and noticed random jerks of the extremities also during sleep, which would awaken the patients at times. Electrolytes were stable with a sodium of 140 on the first day of myoclonic jerking with potassium normal at 3.8 , calcium normal at 8.9, and magnesium normal at 2.10. A non-contrast computed tomography of the head was negative (Figure 1). Past medical history was negative for any neurological problems, and there was no personal or family history for seizures or myoclonic jerks. Neurological exam revealed the patient to be fully oriented with accurate 4 of 4 complex object recall at 5 minutes, with normal deep tendon reflexes and normal muscle tone and strength. The patient reported complete cessation of the myoclonic jerks after discontinuation of antibiotics, and has been free of this problem afterwards.

\section{Discussion}

The presented case clearly illustrates the potential for patients to develop myoclonic jerks as a secondary adverse effects of piperacillin and/or nafcillin. Given the underlying pathophysiology of interaction of penicillin with the gamma-aminobutyric acid type A (GABA-A) receptor chloride channel, it is likely that these two antibiotics may interact in a similar way, and possibly reduce inward inhibitory chloride fluxes into both spinal and supra-spinal neurons. Piperacillin has been noted to not only induce an encephalopathy, ${ }^{1}$ but can also produce seizures as shown by Lin et $a l .{ }^{2}$ who rapidly reversed this by high-flux hemodialysis in a 57 year old end stage renal disease patient who developed 2 generalized tonic-clonic seizures after five $250 \mathrm{mg}$ doses of piperacillin/tazobactam

An excellent study by Sugimoto et al.
Correspondence: Michael Andrew Meyer, Department of Neurology, Sisters Hospital, 2157 Main Street, Buffalo, NY 14214, USA.

Tel.: + 1.716.862.2750.

E-mail: michaelandrewmeyer@gmail.com

Key words: myoclonus, antibiotics, gammaaminobutyric acid, nafcillin, computed tomography.

Conflict of interests: the author declares no potential conflict of interests.

Received for publication: 12 February 2014. Accepted for publication: 4 April 2014.

This work is licensed under a Creative Commons Attribution NonCommercial 3.0 License (CC BYNC 3.0).

(C) Copyright M.A. Meyer, 2014

Licensee PAGEPress, Italy

Neurology International 2014; 6:5349

doi:10.4081/ni.2014.5349

defined the pathophysiology of the ability of penicillin to produce seizure activity by reducing chloride currents across the GABA-A receptor gated channel. ${ }^{3}$ By inducing a selective mutation of tyrosine to phenylalanine at position 256 within the gene encoding the GABA-A receptor gated channel, the charge properties of the central pore region could be changed.

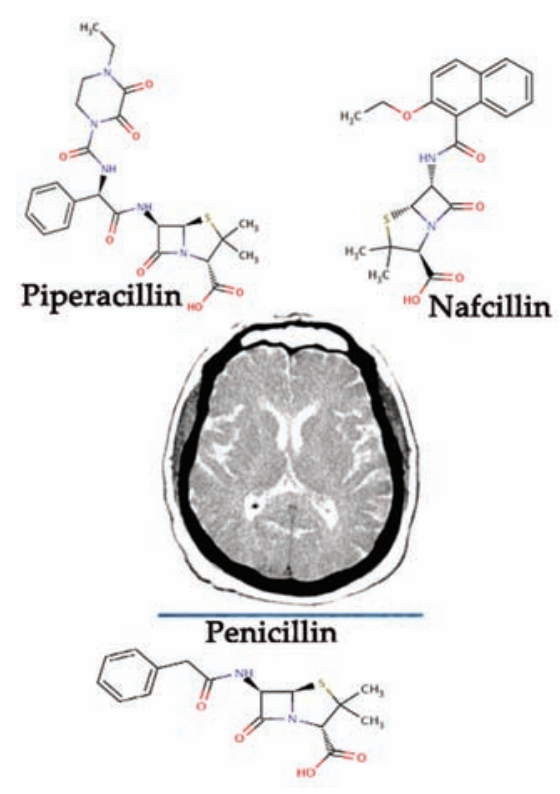

Figure 1. Axial non-contrast head computed tomography is remarkable only for age related atrophy; the structural similarities for piperacillin and nafcillin to penicillin is shown as penicillin is well known to have epileptogenic properties. 
This selective mutation of the beta 2 subunit rendered the altered GABA-A receptor insensitive to the pro-convulsant medication picrotoxin. Detailed voltage clamp study on frog oocytes transfected with these altered channels showed a dramatic inability for penicillin to reduce inhibitory currents.

\section{Conclusions}

In summary, clinicians need to be aware of the potential for triggering myoclonic activity with piperacillin and nafcillin. As they are structurally similar to penicillin, these two antibiotics may place patients at risk as well for developing seizure activity. 4 The pathophysiology for these effects appear to be related to reducing inhibitory hyper-polarizing chloride currents across GABA-A receptor gated channels within the brain

\section{References}

1. Park-Matsumoto YC, Tazawa T. Piperacillininduced encephalopathy. J Neurol Sci 1996; 140:141-2.
2. Lin CS, Cheng CJ, Chou CH, Lin SH. Piperacillin/tazobactam-induced seizure rapidly reversed by high flux hemodialysis in a patient on peritoneal dialysis. Am J Med Sci 2007;333:181-4.

3. Sugimoto M, Fukami S, Kayakiri H, et al. The beta-lactam antibiotics, penicillin-G and cefoselis have different mechanisms and sites of action at GABA(A) receptors. Br J Pharmacol 2002;135:427-32.

4. Fernández-Torre JL, Santos-Sánchez C, Pelayo AL. De novo generalised non-convulsive status epilepticus triggered by piperacillin/tazobactam. Seizure 2010;19: 529-30. 